\title{
Traduire
}

Revue française de la traduction

$239 \mid 2018$

Traduire l'art

\section{Bandes dessinées : le double défi de la langue et de la culture}

Lamia Khelil

\section{(2) OpenEdition}

1 Journals

\section{Édition électronique}

URL : http://journals.openedition.org/traduire/1586

DOI : 10.4000/traduire. 1586

ISSN : 2272-9992

\section{Éditeur}

Société française des traducteurs

\section{Édition imprimée}

Date de publication : 1 décembre 2018

Pagination : 87-94

ISSN : 0395-773X

\section{Référence électronique}

Lamia Khelil, «Bandes dessinées : le double défı de la langue et de la culture », Traduire [En ligne],

239 | 2018, mis en ligne le 01 décembre 2018, consulté le 29 juin 2019. URL : http://

journals.openedition.org/traduire/1586 ; DOI : 10.4000/traduire.1586 


\section{Bandes dessinées : le double défi de la langue et de la culture}

\section{Lamia Khelil}

La bande dessinée et le manga, baptisés neuvième art, ont réussi à s'imposer et à se faire accepter en tant qu'œuvres littéraires à part entière. Mêlant textes et dessins, abordant divers thèmes tels que la jeunesse, l'histoire ou l'humour, cet art pluriel et dynamique a de beaux jours devant lui ! Avec l'avènement de l'internet et grâce à la traduction, il a atteint les contrées les plus lointaines, dans les langues parlées localement.

Nous avons ainsi choisi d'évoquer la traduction de la bande dessinée en langue arabe, avec toutes les richesses linguistiques, grammaticales et culturelles qu'elle véhicule. Si la bande dessinée est l'œuvre de deux artistes, un auteur et un dessinateur, la traduction est l'œuvre d'un seul artiste, le traducteur. Aussi, alors même que l'image reste toujours la même, le texte prend quelquefois dans la langue cible d'autres formes et d'autres tournures afin de transmettre le message au lecteur dans la langue d'arrivée.

Nous allons aborder la traduction de Tintin en langue arabe et nous intéresser à la transposition des onomatopées, interjections, expressions idiomatiques et à toutes ces particularités de la $\mathrm{BD}$; ce sont elles en effet qui véhiculent le génie d'une langue et font la spécificité d'une culture. Comment cette langue et cette culture sont-elles transmises à l'autre ? Comment cet autre les perçoit-il ?

À cet effet, nous avons choisi deux albums de Tintin en langue française et leur traduction en langue arabe, Le crabe aux pinces d'or, devenu Tintin wa al makhaleb edhahabia (littéralement, Tintin et les pinces d'or) et Le lotus bleu, Zahrate al loutus azzarqaa (littéralement, la fleur de lotus bleu).

\section{Résumé des deux albums choisis}

Depuis la création de son personnage en 1929, Tintin a permis aux lecteurs de ses multiples aventures de découvrir le monde et nombre de ses mystères. Ses voyages offraient tout loisir 
d'aller vers l'inconnu sans avoir à quitter sa chambre. Malgré les années, le jeune reporter continue à susciter le même engouement et le même enthousiasme chez ses lecteurs et fans sur toute la planète.

Parmi ses albums, au nombre de vingt-quatre, nous avons opté pour Le lotus bleu, cinquième de la série, dont la version actuelle a été réalisée en 1946. Cet album a été remarqué pour l'abondance des faits historiques présentés, relatifs à la Chine des années 1930. Tout au long de l'aventure, le lecteur est transporté dans ce pays alors envahi par le Japon. II découvre Shanghai, carrefour du commerce international, les fumeries d'opium et les inondations du Yang-Tseu-Kiang qui provoquent des milliers de morts. Dans cet album, Patrick Mérand précise que "Hergé a pris position très très vite dans le conflit sino-japonais, si on regarde bien, les Japonais ont toujours les dents qui dépassent ! "

Le second opus choisi est Le crabe aux pinces d'or, neuvième aventure de Tintin, publiée en 1940-41. Cet album, dont la version couleurs a paru en 1943, marque l'arrivée du capitaine Haddock, personnage emblématique des aventures du jeune reporter. En peu de temps, le marin en vient à occuper une place importante dans les aventures de Tintin grâce à son langage singulier truffé des injures les plus improbables. Ce langage trivial peu soigné, qui fait les délices de bien des amateurs de bande dessinée, causera sans aucun doute quelques soucis aux traducteurs, quelle que soit la langue vers laquelle ils traduisent : ses insultes sont un mélange de vocabulaire marin, de dialecte bruxellois ou tout simplement une sorte de création complexe qui décontextualise les mots pour leur donner un sens nouveau.

\section{Traduire la bande dessinée}

La bande dessinée est une suite d'images et de textes courts qui constitue un discours à forte dominante visuelle mettant à rude épreuve la compétence traductionnelle. Dans son ouvrage Traduire l'image dans les albums d'Astérix. À la recherche du pouce perdu en Hispanie, Yuste Frias précise que

dans toute traduction spécialisée de textes à images, le traducteur ne traduit jamais d'une langue à une autre, isolées de tout autre code sémiotique [...]. Le traducteur doit lire, interpréter et traduire non seulement tous les éléments textuels, mais aussi tous les éléments paratextuels qui composent l'imaginaire présent dans chaque entité iconotextuelle formée par le couple image/texte.

Chaque culture a ses codes sémiotiques et symboliques qu'il convient de prendre en considération du fait de leur spécificité. Le traducteur doit opérer en fonction de la culture réceptrice et des attentes du lectorat.

Dans le cas qui nous intéresse, il doit être attentif non seulement au texte, mais aussi à l'image, car ces deux éléments forment un tout indissociable : de même que le texte, l'image doit être 
lue, interprétée et traduite. Serge Tisseron stipule d'ailleurs que " l'image, bien avant de signifier quelque chose, est un espace ouvert qui nous invite à y entrer et y flâner ".

Le traducteur devra donc opter pour une stratégie qui consiste soit à amener le lecteur vers le texte original et sa culture et à lui communiquer les impressions qu'il aurait eues en lisant l'original, soit à penser la traduction de façon à faire de la culture cible la culture de l'original.

La traduction des bandes dessinées est souvent qualifiée de traduction sous contrainte, du fait de la présence d'onomatopées et de l'espace restreint des bulles. Le traducteur, même s'il est appelé à apporter des modifications au texte, ne saurait remanier l'image, qui demeure inaltérable quelle que soit la stratégie traductionnelle adoptée. Aussi pouvons-nous avancer que l'image est l'obstacle insurmontable qui servira de rempart aux égarements sémantiques du traducteur.

\section{Traduire les onomatopées}

L'une des contraintes majeures que rencontre le traducteur de bande dessinée, notamment le traducteur en langue arabe, est le traitement des onomatopées. II se retrouve devant de multiples choix : doit-il les garder telles quelles, les transcrire phonétiquement en arabe, proposer un équivalent ou simplement les supprimer? Dans les deux albums retenus, nous avons constaté que le traducteur opte indifféremment pour ces diverses alternatives.

Pour les onomatopées qui sont reprises en l'état, par le truchement d'une transcription phonétique en arabe, nous citerons deux exemples. Dans le premier, Toc toc toc, traduit par Toq toq (Le lotus bleu), le traducteur s'est contenté de redoubler la forme Toq afin de rendre les trois toc. Nous supposons que le son émis lorsqu'on frappe à la porte est le même dans toutes les langues. Dans le second, Tchouuum, traduit par Tchoum (Le lotus bleu), on remarque que l'onomatopée française, qui renvoie à l'éternuement, comporte la réplétion de la lettre " u " qui, selon le code sonore français, indiquerait un son grave et prolongé. À ce sujet, Bernard Ducourant invite à

[noter] que les voyelles " 0 " et " u ", redoublées ou non, évoqueront toujours des sons plutôt graves, étouffés, creux, mous (Pof, Boum, Oooh, etc.), tandis que le " $i$ " suggérera au contraire des sons aigus, métalliques (Ziiip) sinon stridents (Hiiiii ou Driiiiin, etc.). Quant au " a ", il produira généralement des sons intermédiaires secs, non métalliques (Clac, Clap, Craaaaac, etc.).

La transcription en arabe ne fait ressortir ni la particularité de ce son ni sa durée. L'équivalent choisi est plutôt très bref et sec: Tchoum. II importait au traducteur de rapporter au lecteur arabophone l'action ayant engendré ce son, qui est l'éternuement, plutôt que l'état de celui qui le produisait.

Pour les équivalents ou la suppression, nous avons choisi des exemples revenant dans les deux albums. Wouah wouah, qui reproduit les aboiements, est traduit en arabe par Haouw 
haouw ; cette transcription en alphabet latin n'est, graphiquement, qu'une simple inversion de lettres permettant d'obtenir en arabe un son nouveau qui renvoie à un même référent.

Dans un autre cas, Pan, qui imite le son produit par un coup de feu, le traducteur a choisi l'équivalent Takh, déjà employé dans une autre bulle pour exprimer un coup de feu qui n'est pas parti (click en français). En l'absence d'image, le lecteur ne comprendrait pas si l'arme a fait feu ou non, le traducteur ayant eu recours au même équivalent pour exprimer deux situations différentes. Le lecteur qui n'a pas sous les yeux la version originale n'y prête pas attention, puisque l'image complète ce manque d'information. Dès lors, nous pouvons avancer que le traducteur a rendu les sons par d'autres sons équivalents ou identiques en essayant de garder au maximum les onomatopées de base.

\section{Traduire les interjections}

Il est difficile de séparer les interjections des onomatopées, ces deux concepts étant étroitement imbriqués ; on les confond d'ailleurs, certaines onomatopées pouvant être des interjections. Cependant, Sierra Soriano Ascension postule que

pour obtenir une bonne traduction de $B D$, il est évident [...] qu'il convient de connaître à fond les différentes valeurs de l'interjection et ses différents emplois dans la langue parlée française [...] II semble que traduire une BD consiste surtout à traduire des interjections ou, tout au moins, que la traduction des interjections constitue un énorme travail et une partie significative dans le processus de traduction d'une $B D$.

Aussi avons-nous tenté de cerner des interjections qui ne prêtent pas à confusion. Nous avons sélectionné celles suivies d'un point d'exclamation, des locutions interjectives et des interjections répétées.

* Pour l'interjection " simple ", nous avons choisi dans Le lotus bleu l'exemple Psst ! (son bref émis pour interpeller quelqu'un discrètement), traduit par Ahlan! (littéralement, bienvenue).

* Pour la locution interjective, nous avons opté pour Psst ! Hey ! psst ! (Le crabe aux pinces d'or), traduit par Tintin Tintin! inana houna taala (littéralement : Tintin, Tintin ! Viens, on est là).

La traduction proposée pour ces deux exemples nous a intéressés pour plusieurs raisons. L'interjection choisie n'a en effet pas la même valeur dans les deux langues. Alors qu'elle n'est qu'une simple façon d'interpeller un individu dans la langue française, elle correspond à une apostrophe plutôt particulière en arabe (du moins dans les pays du Maghreb) : c'est le moyen qu'utiliserait un jeune homme pour attirer l'attention d'une jeune fille dont il souhaiterait faire la connaissance. 
Le traducteur, afin de ne pas heurter certaines susceptibilités et sensibilités, est ici contraint d'effectuer une adaptation acceptable dans la culture d'arrivée. La traduction propose ainsi le plus conventionnel Ahlan! qui ne cadre cependant pas avec l'image, où l'on voit les personnages interpellés se retourner avec un air menaçant. On souhaite en effet la bienvenue en faisant face à la personne que l'on accueille, plutôt qu'en l'interpellant par-derrière. Le traducteur a préféré ne pas traduire l'interjection par une autre interjection, même s'il a gardé le point d'exclamation. Le lecteur en déduira par lui-même que les personnages hélés par Tintin ne sont pas forcément des amis.

Pour le second exemple, l'expression interjective Psst ! hey ! psst !, le traducteur, au lieu de chercher dans la culture d'arrivée une interjection appellative équivalente, a préféré modifier le texte et a tenté de rendre le sens au moyen d'une autre structure syntaxique, qui peut alors être considérée comme un texte explicatif. II a déduit le sens du texte à l'aide de la bulle suivante, d'où l'ajout de Tintin, Tintin ! qui n'existe pas dans le texte original. En effet deux personnages interpellent Tintin en lui faisant signe avec leurs cannes, pour lui signifier qu'ils sont juste à côté et qu'il peut les rejoindre - ce que l'on retrouve dans la suite de la traduction en arabe, inana houna taala (Viens, on est là). Le traducteur estime que l'on ne saurait interpeller quelqu'un dans la rue par un simple Psst dans la culture cible ; étant donné que l'on connaît cette personne, on doit l'appeler par son nom. De cette façon, le texte traduit reste tout aussi expressif et paraît naturel au lecteur arabe.

II arrive aussi que le traducteur fasse l'impasse sur certaines interjections, Driiing par exemple, qui annonce qu'on sonne à la porte ; on voit Tintin se retourner pour identifier l'origine de cette sonnerie. Or dans la version arabe, le dessin est le même, sans que l'on comprenne d'abord la raison du mouvement de Tintin. La réponse sera fournie par l'image suivante, qui montre l'arrivée du facteur avec du courrier : le dessin a remplacé le texte pour la transmission du sens.

\section{Traduire les expressions idiomatiques}

Alain Rey affirmait que :

Parmi les éléments de la langue qu'il faut acquérir pour s'exprimer figurent non seulement des mots, mais aussi des groupes de mots plus ou moins imprévisibles, dans leur forme parfois, et toujours dans leur valeur. Ainsi des milliers de particularités expressives, non traduisibles telles quelles dans d'autres langues, forment l'immense part du lexique connu sous le nom d'expressions idiomatiques.

La langue française recèle d'innombrables expressions idiomatiques. Le traducteur du français vers une autre langue aura le choix entre chercher dans la langue d'arrivée une expression équivalente apte à rendre le même sens, ou tenter de la signifier sans l'emploi d'un idiotisme, chose qui n'est pas toujours aisée. 
Dans l'album Le crabe aux pinces d'or, nous avons relevé l'expression Quelle mouche l'a piqué ?, traduite par toura ma al ladhi hadatha likay yakhroudja bi hadhihi essouraa! (littéralement : Qu'est-ce qui a bien pu se passer pour qu'il sorte à cette vitesse !). Dans cette formule qui marque la surprise et l'étonnement quant à la réaction soudaine de l'un des personnages, le traducteur, n'ayant pas trouvé d'expression équivalente, a tenté d'expliciter le propos en transformant l'interrogation en exclamation. Cela permet de transmettre l'idée tout en conservant l'étonnement et la surprise qui ressortent de l'original. Le lecteur retrouvera ainsi l'expressivité du texte source dans une version légèrement plus détaillée.

Pour reproduire l'expression mettre aux fers, traduite par sadhaouka fi al hadid (littéralement : Je te mettrai aux fers), le traducteur procède cette fois par une traduction littérale, qui n'est même pas sémantique. En introduisant dans la langue cible une expression qui n'existe pas, il ne peut créer un effet similaire à l'original, et le lecteur ne saisira pas immédiatement l'intention de l'auteur.

Par ailleurs, dans l'album Le lotus bleu, nous avons relevé la tournure Nous sommes passés comme une lettre à la poste, rendue par laqad mararna bi salam (littéralement : On est passé en paix). En l'absence d'expression similaire en langue arabe, le traducteur a concentré ses efforts sur le côté sémantique. Aidé de l'image, le lecteur pourra aisément saisir le sens ; la traduction a donc produit le même effet et transposé le soulagement que reflètent les visages des personnages.

\section{Conclusion}

Nous pouvons en conclure qu'il n'est pas impossible de traduire la bande dessinée et tous les éléments qui la composent, la présence de l'image servant de guide au traducteur et lui permettant de systématiquement trouver une parade. Ce même traducteur ne va pas s'attarder sur la signification des mots prononcés par chacun des personnages, il va davantage axer son travail sur l'énoncé dans son ensemble.

En tant que passeur entre deux langues et deux cultures très différentes, le traducteur sera confronté à deux choix : soit adapter le texte source à la culture cible, soit trouver le meilleur moyen pour faire accepter la culture de l'autre sans toutefois heurter les sensibilités de quiconque.

Imkhelil@gmail.com

Lamia Khelil est maître de conférences à l'Institut de Traduction, Université d'Alger 2. Elle enseigne la traduction spécialisée de l'arabe au français et du français à l'arabe. 


\section{Bibliographie}

BLIN Louis, 2015, Le monde arabe dans les albums de Tintin, Paris, L'Harmattan.

DUCOURANT Bernard, 1982, L'Art de la B.D, Grenoble (France), Éditions Glénat, Tome 1.

HERGÉ, 1952, Les aventures de Tintin : Le lotus bleu, Tournai (Belgique), Casterman.

HERGÉ, (Année inconnue), Moughamarate mouthira tintin, zahratou al loutus al zarqaa, Le Caire (Egypte), traduit aux éditions Dar Al Maarif, traducteur inconnu

$$
\text { هيرجي، مغامرات مثيرة تانتان و زهرة اللوتس الزرقاء، القاهرة ( مصر)، ترجمة دار المعارف للطبعة العربية }
$$

HERGÉ, 1960, Les aventures de Tintin : Le crabe aux pinces d'or, Belgique, Casterman.

HERGÉ, 1998, Moughamarate mouthira, tintin wa al makhalib edhahabia, Le Caire (Egypte), traduit par les éditions Dar Al Maarif, 4e édition, traducteur inconnu.

هيرجي، 1998، مغامرات مثيرة تانتان و المخالب الذهبية، القاهرة ( مصر )، ترجمة دار المعارف للطبعة العربية

MERAND Patrick. Li Xiaohan, 2009, Le lotus bleu décrypté, Saint-Maur-des-Fossés (France), Sépia.

REY Alain. CHANTREAU Sophie, 1979, Dictionnaire des expressions et locutions, Éd. du Robert SIERRA SORIANO Ascension, 1999, "L'interjection dans la BD : réflexions sur sa traduction ", in Meta, 44(4), p. 582-603, consulté le 29/09/2018.

TISSERON Serge, 2003, Le bonheur dans l'image, Paris, Les Empêcheurs de penser en rond.

VELEZ Antonio, 2014, "Jurons et interjections dans l'univers de Tintin : Modifications, élargissement et réduction de sens dans les traductions italiennes ", in Studia UBB Philologia, 59(3), p. 25-43.

YUSTE FRÍAS José, 2011, "Traduire l'image dans les albums d'Astérix. À la recherche du pouce perdu en Hispanie ", in RICHET B. (éd.), Le tour du monde d'Astérix, Actes du colloque tenu à la Sorbonne les 30 et 31 octobre 2009, Paris, Presses Sorbonne Nouvelle. 


\section{Webographie}

KHOURY Georges (Jad), La bande dessinée d'expression arabe de 1950 à nos jours, Takam Tikou,

http://takamtikou.bnf.fr/dossiers/dossier-2011-la-bande-dessinee/la-bande-dessinee-dexpression-arabe-de-1950-a-nos-jours, consulté le 25/09/2018.

RIVIÈRE Juliette, 2018, La bande dessinée : le 9e art, Kazoart, https://www.kazoart.com/blog/la-bande-dessinee-le-9e-art/, consulté le 15/05/2018.

http://www.tintin.free.fr/galerie/personnages.php?choix=haddock, consulté le 24/09/2018. 